\title{
Examination of the Common Cause Account in a Population-Based Longitudinal Study with Narrow Age Cohort Design
}

\author{
Ola Sternäng ${ }^{a, b}$ Bert Jonsson ${ }^{c}$ Åke Wahlin ${ }^{a, b}$ Lars Nyberg $^{d}$ \\ Lars-Göran Nilsson ${ }^{\mathrm{a}, \mathrm{b}}$

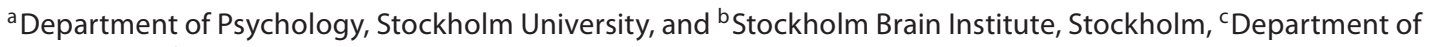 \\ Psychology, dDepartment of Integrative Medical Biology and Department of Radiation Sciences, \\ Umeå University, Umeå, Sweden
}

\section{Key Words}

Common cause account $\cdot$ Sensory functioning $\cdot$ Cognitive functioning

\begin{abstract}
Background: The common cause account suggests that there is a third factor causing aging effects in both sensory and cognitive functioning, hypothesized to be the integrity of the central nervous system [Lindenberger and Baltes; Psychol Aging 1994;9:339-355]. Importantly, the common cause account was developed based on cross-sectional data, which are especially biased by cohort effects. However, cohort effects can be controlled for in narrow age cohort (NAC) designs and by longitudinal examination. Findings from the few longitudinal studies that have studied the relation between age-related changes in sensory and cognitive functions are complex and give only partial support to the common cause account. Objective: The present paper examines the common cause account within a longitudinal setting. Method: Our study is unique in the sense that it tests the common cause account within a longitudinal NAC design using data from the Betula project. The participants $(n=$ $1,057)$ were in the age range of $45-90$ years. Results: The findings indicate that the relationship between sensory and memory functioning in both a longitudinal age-heteroge-
\end{abstract}

neous and a longitudinal NAC design are much weaker than that detected by an age-heterogeneous cross-sectional design. Conclusion: The demonstrated weak age-associated sensory-cognitive link raises questions regarding the explanatory value of the common cause account and related theoretical accounts for accounting for age-related cognitive changes.

Copyright $\odot 2010$ S. Karger AG, Basel

Within the field of cognitive aging, there are established meditational accounts for age-related cognitive decline (for a review, see [2]). Candidate mediators such as processing speed, executive abilities and sensory/sensorimotor functions have been put forward as explanatory variables for age-related variance in cognitive tasks [2]. Initially, Lindenberger and Baltes $[1,3,4]$ identified sensory/sensorimotor functioning as a possible mediator explaining age-related variance in cognitive abilities. Several other cross-sectional studies have found similar results when examining the age-related connection between sensory/sensorimotor and cognitive functioning (see [5] for a review). Furthermore, this association also seems to be stronger in samples of older persons $[4,6]$.

The causal relation between sensory/sensorimotor abilities and age-related cognitive decline is not clear, but

\section{KARGER}

Fax +4161306 1234 E-Mail karger@karger.ch www.karger.com

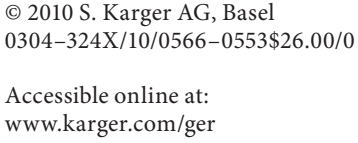


according to Baltes and Lindenberger [4], the most plausible explanation is the so-called common cause account, i.e. there is a common factor that affects both cognition and sensory/sensorimotor functioning. This common factor has been hypothesized to be the functioning of the central nervous system in a very broad sense [1]. Thus, a depleting common neurophysiological resource leads to simultaneous decline in both cognitive and sensory/sensorimotor functioning.

However, the studies cited above have used a crosssectional approach, a design criticized not only for the influence of cohort effects, but also for producing spurious correlations due to population mean trends $[5,7]$. Therefore, the associations can simply be based on mean trends and are, in that case, unrelated to true age-related processes. An alternative to the common cross-sectional design is a cross-sectional design studying associations within narrow age cohorts, a so-called narrow age cohort (NAC) design [5, 7-9]. By studying age-homogenous cohorts, time is held constant. The NAC design makes it possible to investigate the effects of aging (age-related changes) without the possible bias due to population mean trends $[7,8]$.

There are only a few studies that have used NAC design, and those have, in comparison to age-heterogeneous samples, found relatively weak relations of cognitive and sensory/sensorimotor variables. For example, Hofer et al. [5] found a moderate association between visual acuity and cognitive measurements and no association of hearing with cognitive measures. They suggested that, instead of a general central sensory decline, specific peripheral losses probably affect specific cognitive abilities. Tay et al. [10] examined four age groups above 50 years of age and found weak but significant correlations between sensory and cognitive functioning for participants without sensory or cognitive impairment.

Examination of individual aging effects across time is likely to show that these are stronger in older cohorts. The fact that persons differ in their rates of aging will likely also result in more variation the longer persons are removed from their early adulthood levels [5]. Interindividual variation within the same age group also seems to increase across increasing age [11].

It is important to stress that a cross-sectional NAC design is still cross-sectional and cannot say much about causality. Aging is a process that goes on within individuals and is in that sense best studied with longitudinal design [5]. Admittedly, longitudinal studies have shortcomings, such as attrition and re-test effects, but attrition problems also exist in cross-sectional studies and are probably more dangerous since they are not directly observable [8]. Findings from the few longitudinal studies that have examined the relation between age-related changes in sensory/sensorimotor and cognitive functions are complex and give only partial support to the common cause account [12-15]. Based on these kind of findings, $\mathrm{Li}$ and Lindenberger [13] suggested that a common factor might not be the only cause, and that it is likely that there are also unique effects that (to various degrees) underlie sensory and cognitive decline.

The present study is unique in the sense that it combines a sequential NAC design with a longitudinal design [9] to examine the common cause account. Data from the Betula project $[16,17]$ were used. Betula is a longitudinal population-based cohort study, with the main aim to evaluate the development of memory functions and health in adults aged 35 years and older.

Our hypotheses were (1) that associations between memory and sensory functioning are stronger when the age of the participants in a sample varies widely (and therefore includes population mean trends, as opposed to when age is held constant), (2) these associations are stronger in cross-sectional than in longitudinal data, and (3) stronger in older than in younger age cohorts.

\section{Method}

\section{Participants}

The participants were recruited from the ongoing Betula project, which is a longitudinal population-based study that started in 1988-1990 (time 1; T1). After this first wave of data collection, another three waves of data collection were administrated: Time 2 (T2) in 1993-1995, time 3 (T3) in 1998-2000 and time 4 (T4) in 2003-2005. The research protocols in Betula have been fully described elsewhere $[16,17]$. Since relevant sensory measures were not included in the Betula project until T3, the present study used data from T3 and T4. The participants (age range 45-90 years) were recruited from two independent samples: sample 1 (S1; T3 was their third test occasion), and sample 3 (S3; T3 was their second test occasion). The total number of participants was 1,966.

In order to study normative aging, participants diagnosed with dementia at T2, T3 or T4 $(\mathrm{n}=182)$ were excluded from the study. Participants that did not participate at either T3 or T4, and hence could not be diagnosed, were also excluded $(n=727)$. This resulted in a final sample of 1,057 persons: 580 women and 477 men. A description of the final sample and participants excluded from the study is provided in table 1.

Measures

During each of the data-collection waves, the sensory and cognitive data were collected at two separate occasions that lasted between 1.5 and $2 \mathrm{~h}$ each (see [16,17] for detailed descriptions of the procedure in Betula). 
Table 1. Description of participants included in and excluded from the study

\begin{tabular}{|c|c|c|c|}
\hline \multirow[t]{2}{*}{ Variable (at T3) } & \multicolumn{2}{|c|}{ Excluded from the sample } & \multirow{2}{*}{$\begin{array}{l}\text { Included in } \\
\text { the sample } \\
(\mathrm{n}=1,057)\end{array}$} \\
\hline & $\begin{array}{l}\text { demented at } \\
\mathrm{T} 2-\mathrm{T} 4 \\
(\mathrm{n}=182)\end{array}$ & $\begin{array}{l}\text { others } \\
(n=727)\end{array}$ & \\
\hline Age & $81.1 \pm 7.3$ & $72.5 \pm 14.3$ & $61.0 \pm 11.6$ \\
\hline Women & $113(62 \%)$ & $384(53 \%)$ & $580(55 \%)$ \\
\hline Education, years & $7.9 \pm 3.5$ & $9.9 \pm 4.3$ & $11.2 \pm 4.2$ \\
\hline Episodic memory ${ }^{\mathrm{a}}$ & $-1.18 \pm 0.65$ & $-0.31 \pm 0.63$ & $0.00 \pm 0.61$ \\
\hline Semantic memory ${ }^{\mathrm{a}}$ & $-1.13 \pm 1.10$ & $-0.44 \pm 0.95$ & $0.00 \pm 0.77$ \\
\hline \multicolumn{4}{|l|}{ Sensory } \\
\hline functioning ${ }^{\mathrm{a}}$ & $-0.57 \pm 0.89$ & $-0.30 \pm 0.96$ & $0.00 \pm 0.73$ \\
\hline
\end{tabular}

Post hoc tests (Scheffé) showed significant differences between all three groups for the variables age, years of education, episodic memory and semantic memory.

${ }^{a}$ Episodic memory, semantic memory and sensory functioning are $\mathrm{z}$-score composites.

\section{Cognitive Variables}

For the purposes of the present study, we focused on processes involving episodic memory (a fluid ability) and semantic memory (a crystallized ability). In order to reduce the number of analyses and increase reliability, cognitive composite scores were constructed. The two dependent variables constructed for the purposes of this study were episodic memory (a composite of 15 indicators) and semantic memory (a composite of 3 indicators). The cognitive composite scores were obtained by summing and averaging across the contributory indicators, of which all were continuous and $\mathrm{z}$ transformed. This $\mathrm{z}$ transformation procedure was done separately both for the total final sample (for the age-heterogeneous analyses) and for every age cohort (for the age-homogeneous analyses). The final sample was divided into 9 age cohorts $(45,50,55,60,65$, $70,75,80$ and $85-90$ years of age at T3). For a description of sex and sample distribution in the different age cohorts, see table 2 .

Table 3 shows an overview of the 18 cognitive indicators used in this study, numbered in the order they were administered. A brief summary of these tests is given below (for a full description of the battery, see $[16,17])$.

Recall and Recognition of Sentences with or without

Enactment at Study (Indicators 2, 3, 4, 5, 11, 12, 13 and 14)

The participants studied 2 lists of 16 short sentences in imperative forms. Each sentence was shown on an index card for $8 \mathrm{~s}$ and consisted of 1 verb and 1 noun (e.g. lift the book, crumple up the tie, point at the apple). The participants were instructed to read aloud the commands. For one list (the subject performed condition), the experimenter handed over an object for each of the 16 sentences (the noun in each sentence) and the participant was instructed to perform the command in connection to the sentence. For the other list (the verbal condition), the participant was shown 16 index cards with the same type of sentences, but no enactment was demanded from the subject.

Sensory and Cognitive Functioning
Table 2. Distribution of sex and samples in the age cohorts and in the total age-heterogeneous sample

\begin{tabular}{|c|c|c|c|}
\hline Age at T3 & Women & Men & Total \\
\hline \multicolumn{4}{|c|}{ Age cohorts } \\
\hline \multirow[t]{2}{*}{45} & 37 (S1) & $33(\mathrm{~S} 1)$ & $70(\mathrm{~S} 1)$ \\
\hline & 42 (S3) & 40 (S3) & $82(\mathrm{~S} 3)$ \\
\hline Total & 79 & 73 & 152 \\
\hline \multirow[t]{2}{*}{50} & $35(\mathrm{~S} 1)$ & $35(\mathrm{~S} 1)$ & $70(\mathrm{~S} 1)$ \\
\hline & $45(\mathrm{~S} 3)$ & 39 (S3) & $84(\mathrm{~S} 3)$ \\
\hline Total & 80 & 74 & 154 \\
\hline \multirow[t]{2}{*}{55} & $34(\mathrm{~S} 1)$ & $30(\mathrm{~S} 1)$ & $64(\mathrm{~S} 1)$ \\
\hline & $36(\mathrm{~S} 3)$ & 39 (S3) & 75 (S3) \\
\hline Total & 70 & 69 & 139 \\
\hline \multirow[t]{2}{*}{60} & $45(\mathrm{~S} 1)$ & $33(\mathrm{~S} 1)$ & $78(\mathrm{~S} 1)$ \\
\hline & $46(\mathrm{~S} 3)$ & $36(\mathrm{~S} 3)$ & $82(\mathrm{~S} 3)$ \\
\hline Total & 91 & 69 & 160 \\
\hline \multirow[t]{2}{*}{65} & $36(\mathrm{~S} 1)$ & $32(\mathrm{~S} 1)$ & $68(\mathrm{~S} 1)$ \\
\hline & 43 (S3) & $25(\mathrm{~S} 3)$ & $68(\mathrm{~S} 3)$ \\
\hline Total & 79 & 57 & 136 \\
\hline \multirow[t]{2}{*}{70} & $28(\mathrm{~S} 1)$ & $32(\mathrm{~S} 1)$ & $60(\mathrm{~S} 1)$ \\
\hline & $38(\mathrm{~S} 3)$ & $25(\mathrm{~S} 3)$ & $63(\mathrm{~S} 3)$ \\
\hline Total & 66 & 57 & 123 \\
\hline \multirow[t]{2}{*}{75} & $24(\mathrm{~S} 1)$ & $16(\mathrm{~S} 1)$ & $40(\mathrm{~S} 1)$ \\
\hline & $30(\mathrm{~S} 3)$ & $21(\mathrm{~S} 3)$ & $51(\mathrm{~S} 3)$ \\
\hline Total & 54 & 37 & 91 \\
\hline \multirow[t]{2}{*}{80} & $14(\mathrm{~S} 1)$ & $15(\mathrm{~S} 1)$ & $29(\mathrm{~S} 1)$ \\
\hline & $18(\mathrm{~S} 3)$ & $9(\mathrm{~S} 3)$ & 27 (S3) \\
\hline Total & 32 & 24 & 56 \\
\hline \multirow[t]{2}{*}{$85-90$} & $14(\mathrm{~S} 1)$ & $5(\mathrm{~S} 1)$ & $19(\mathrm{~S} 1)$ \\
\hline & $15(\mathrm{~S} 3)$ & $12(\mathrm{~S} 3)$ & 27 (S3) \\
\hline Total & 29 & 17 & 46 \\
\hline \multicolumn{4}{|c|}{ Total sample } \\
\hline \multirow[t]{2}{*}{$45-90$} & $267(\mathrm{~S} 1)$ & $231(\mathrm{~S} 1)$ & 498 (S1) \\
\hline & 313 (S3) & 246 (S3) & $559(\mathrm{~S} 3)$ \\
\hline Total & 580 & 477 & 1,057 \\
\hline
\end{tabular}

Immediately afterwards, the participants were given a free recall test of the subject performed task (indicator 2) within a 2-min time frame and $2 \mathrm{~min}$ were also allotted for a free recall test of the verbal task (indicator 3 ) or vice versa with respect to order.

The nouns in the 32 sentences belonged to 8 different categories (e.g. reading materials, clothes and fruits), 4 from each list, with 4 nouns in each. Three minutes were allowed for a cued recall test of enacted and non-enacted sentences (indicator 4 and 5) with the 8 category names serving as retrieval cues for these 32 sentences. This test was administered immediately after the 2 free recall tests and the task was to recall as many correct nouns as possible.

A free-choice recognition test was administered (indicator 11 and 12) approximately $40 \mathrm{~min}$ after the study of the sentences, where the participants were asked to tell whether they recognized 
Table 3. Cognitive indicators

\begin{tabular}{lll}
\hline Cognitive tasks & $\begin{array}{l}\text { Number (in order } \\
\text { of appearance) }\end{array}$ & $\begin{array}{l}\text { Performance score } \\
\text { (number of ......) }\end{array}$ \\
\hline
\end{tabular}

Episodic memory (15 indicators)

Recall (10 indicators)

Free recall (5 indicators)

- of sentences with enactment at study 2.

- of sentences without enactment at study 3 .

- of word list with concurrent task at study 15.

- of word list with concurrent task at test 16.

- of word list with no concurrent task 17.

Cued recall (5 indicators)

- of sentences with enactment at study (category as cue) 4 .

- of sentences without enactment at study (category as cue) 5 .

- of sentences with enactment at study (verb as cue) 13.

- of sentences without enactment at study (verb as cue) 14 .

- of activities during the test session 18.

Recognition (5 indicators)

Free-choice recognition ( 3 indicators)

- of faces 8

- of nouns in sentences with enactment at study 11.

- of nouns in sentences without enactment at study 12.

Forced-choice recognition ( 2 indicators)

- of first names

- of family names

.

...... correct verbs and nouns

...... correct verbs and nouns

...... correct words

...... correct words

...... correct words

...... correct nouns

...... correct nouns

...... correct nouns

...... correct nouns

..... correct activities

Semantic memory (3 indicators)

Word comprehension ( 1 indicator)

Word category fluency ( 2 indicators)

- of five-letter words with the initial letter M

- of names of professions with the initial letter B

...... hits minus number of false alarms

1. ..... hits minus number of false alarms

2. $\quad . . .$. hits minus number of false alarms

9. $\quad$...... hits

10. $\quad$...... hits

All episodic memory indicators assess intentional learning, except indicator 9 and 18 that assess incidental learning.

the nouns from the sentences with or without enactment as described above. The participants were presented, in randomized order, with 16 nouns from the study lists and 16 distracter nouns. The 16 nouns from the study lists comprised 8 nouns from the sentences with enactment and 8 nouns from the sentences without enactment. The distracter nouns belonged to the same semantic categories as the target nouns from the study lists. A maximum of $5 \mathrm{~s}$ per word were allotted. The number of hits minus false alarms was the dependent variable.

About 10 min later, another cued recall test was performed where the verb instead served as a retrieval cue. The task was to recall as many correct nouns as possible and $8 \mathrm{~s}$ per retrieval cue were allowed. This was done both for the subject performed (indicator 13) and the verbal condition (indicator 14).

\section{Recall of Nouns with Concurrent Task (Indicators 15, 16} and 17)

The participants were presented auditorily with 3 lists of 12 common nouns (unrelated) and each noun was presented for $2 \mathrm{~s}$. The concurrent task was to sort a deck of playing cards into red cards and black cards. Three different conditions were associated with the 3 lists, which were: the concurrent task was done only during study (indicator 15), the concurrent task was done only during the test (indicator 16) and no concurrent task was done at all (indicator 17). The number of nouns recalled within $1 \mathrm{~min}$ constituted the main outcome in all 3 conditions.

Memory for Activities (Indicator 18)

Each participant was asked, at the end of the test session, to recall in any order as many of the activities during the test session as possible. No time limit was set for this task.

Face and Name Recognition (Indicators 8, 9 and 10)

This task consisted of a presentation of color photographs of 16 faces of 10 -year-old children together with made-up names. The made-up names were combinations of common Swedish first and family names. Each photograph, with name, was presented for $8 \mathrm{~s}$. Approximately $30 \mathrm{~min}$ later during the session, a test of recognition of faces (indicator 8) was administered, showing 12 'correct' faces and 12 distracter faces. The test of names (indicator 9 and 10) was multiple choice (4 alternatives) with 1 correct name and 3 distracter names. The maximum time allowed for each pho- 
tograph was $15 \mathrm{~s}$. Earlier, the participants were only told to remember the family names. Thus, the remembering of family names was related to intentional learning and remembering the first names constituted incidental learning.

Word Category Fluency (Indicators 6 and 7)

Tests of word category fluency with 2 different tasks, lasting 1 min each, were administered. The first was to generate aloud as many 5-letter words as possible starting with the letter ' $M$ ' (indicator 6), and the second task was to generate aloud names of professions starting with the letter 'B' (indicator 7).

\section{Word Comprehension (Indicator 1)}

A word comprehension test with 30 target words was used. For each target word, another 5 words were presented including 1 synonym to the target word. The task was to underline each synonym and the time allotted was $7 \mathrm{~min}$.

\section{Sensory Variables}

Visual Acuity

A Jaeger eye chart [18] was used for determining near vision (e.g. reading a book or a newspaper). The participants used reading glasses or lenses if they had used glasses or lenses during the other parts of the health examination. The Jaeger eye chart was a hand-held card in A5 format, which the participant held at a convenient reading distance. It contained 8 paragraphs printed with 8 different font sizes, and the participant was asked to read the paragraph with the smallest font size possible. The vision score corresponded to the paragraph with the smallest font-size where the participant could read the whole paragraph. The font size of the paragraphs went from 5 points (lowest row) to 24 points (top row). This test was done separately for the right and left eye, with the participant starting with the right eye. The highest score (worst performance) of the right and left eye performance was used in this study as a total visual acuity measure.

\section{Auditory Acuity}

The auditory measure used was the five minute hearing test (FMHT) developed by the American Academy of Otolaryngology - Head and Neck Surgery. One study of the validity [19] has showed that the FMHT is significantly correlated with several standard audiological measures, such as pure tone audiometry. FMHT is a questionnaire that consists of 15 questions, with the score on each item corresponding to a 4 -point Likert scale (almost always -3 , half the time -2 , occasionally -1 , never -0 ). The questions regarded how the participant perceived his/her hearing difficulty in different settings (e.g. when talking on the phone, watching TV and in noisy situations). The auditory measure used in the present study was the total test score.

\section{Sensory Composite}

In order to calculate a sensory composite, $\mathrm{z}$ transformation of the two sensory measures above was made. The composite consisted of the average of the two z-transformed sensory measures (both measures was multiplied by -1 , since both hearing and vision scores were higher the worse the participants were able to hear or see).

\section{Independent and Dependent Variables}

Episodic and semantic memory constituted the dependent variables in the study, while sensory functioning and chronological age served as independent variables. Sex and years of education were selected as general descriptive variables.

\section{Statistical Analyses}

All statistical analyses (except the latent difference score analyses, see below) were performed using SPSS statistical software package (version 11.0 for Mac OS X). Alpha levels were set at $\mathrm{p}<$ 0.05 . Missing cognitive and sensorimotor data (range: $0-5 \%$ ) were replaced using the estimation maximization method, which is known to create realistic approximations of variance [20].

In order to examine age-related variation, we partitioned the cognitive variation associated with chronological age, to examine the extent to which sensory functioning accounted for this variation. This partitioning was done through hierarchical regression where sensory functioning was entered first and chronological age in a second step. The formula used was $a=(b-c) / b$, where $\mathrm{a}=$ explained age-related variance, $\mathrm{b}=$ the amount of variance that age explains in the cognitive variable (i.e. episodic or semantic memory) if entered alone in a regression model, and $c=$ the amount of variance that age explains in the cognitive variable, if sensory functioning is entered first and chronological age second in a hierarchical regression.

However, hierarchical regression is a correlational method and as such it has its limitations [21]. Lindenberger and Pötter [21] showed that the results can be influenced by the age-partialed correlation between the mediator and the dependent variable. They pointed out that it is important that a mediation assumption is based on theory and not only on results from a hierarchical regression.

For the age-heterogeneous longitudinal analyses, both the different scores and latent difference scores (LDS) [22-24] were used. LDS analysis is a structural equation approach of analyzing longitudinal data. It integrates other structural equation models, such as latent growth and autoregressive models, which makes it rather flexible. Instead of manifest difference variables, the LDS approach uses latent difference variables calculated from the underlying structural relationships.

In the present study, bivariate LDS models were used in order to estimate LDS correlations between memory and sensory functioning. This type of model has been described well by McArdle $[22,23]$ and McArdle and Hamagami [24], and in the present study this model was performed in its simplest form with 2 time points only. The LDS calculations were made with LISREL (version 8.80), and as an index of close fit for the LDS models, the probability that the root mean square error of approximation [25] was below 0.05 was used.

\section{Results}

Two different designs were used: a cross-sectional (at T3) and a longitudinal study over 5 years (from T3 to T4). Two ways of analyzing longitudinal change were used in this section: difference scores and LDS. Both the crosssectional and the longitudinal analyses with difference 
scores were conducted in two steps. First, correlations between the cognitive measures, chronological age and sensory functioning were calculated for the age-heterogeneous sample. Hierarchical regression analyses were made in the cross-sectional design to examine the amount of explained age-related variance. Second, correlations between the cognitive measures and the sensory variable were examined in a sequential NAC design. Besides the calculations (described above) with difference scores, LDS analyses were performed in the age-heterogeneous longitudinal sample in order to calculate the LDS correlations between memory and sensory abilities.

In this section, three main terms are used: correlation between two variables ( $r$ ), explained or shared variance $\left(r^{2}\right)$ and explained age-related variance (see previous paragraph for how explained age-related variance was calculated).

\section{Cross-Sectional Analyses}

Age-Heterogeneous Sample

The correlations and explained variances for the ageheterogeneous sample $(n=1,057)$ are shown in table 4 . The correlations for episodic and semantic memory with sensory functioning were $r=0.33$ and $r=0.26$, respectively. The explained variances for both episodic and semantic memory with sensory functioning were, therefore, rather low (11 and 7\%, respectively). Note that these estimates are based on an age-heterogeneous sample and the underlying correlations might differ across age cohorts. Correlations across cohorts were analyzed in the NAC samples section below.

The explained age-related variance by the sensory variable was $29 \%$ for episodic memory and $42 \%$ for semantic memory (see table 5). A division of the sample in a young (45-65 years of age) and old group (70-90 years of age) was done, and identical analyses in the two groups showed that the corresponding figures for the old group were higher (41 and 55\%, respectively) than for the young group (20 and $36 \%$, respectively).

\section{NAC Samples}

The correlations and explained variances in the NAC samples are shown in table 6 . The age cohort correlations of episodic memory with sensory functioning varied in the range between $r=-0.02$ and $r=0.49$, with a weighted average of $r=0.14$. The weighted average was weighted according to sample size. The corresponding range for the cohort correlations between semantic memory and sensory functioning was rather similar: between $\mathrm{r}=$ -0.07 and $r=0.52$, with $r=0.13$ for the weighted aver-
Table 4. Correlations and explained variance in the age-heterogeneous sample $(\mathrm{n}=1,057)$

\begin{tabular}{|c|c|c|c|c|c|c|}
\hline \multirow[t]{2}{*}{ Cross-sectional (at T3) } & \multicolumn{3}{|c|}{$\begin{array}{l}\text { vs. sensory } \\
\text { functioning }\end{array}$} & \multicolumn{3}{|c|}{ vs. age } \\
\hline & $\mathrm{r}$ & & $r^{2}$ & $\mathrm{r}$ & & $\mathrm{r}^{2}$ \\
\hline Episodic memory & 0.33 & $* *$ & 0.11 & -0.56 & ** & 0.31 \\
\hline Semantic memory & 0.26 & $* *$ & 0.07 & -0.33 & ** & 0.11 \\
\hline Sensory functioning & & & & -0.36 & ** & 0.13 \\
\hline
\end{tabular}

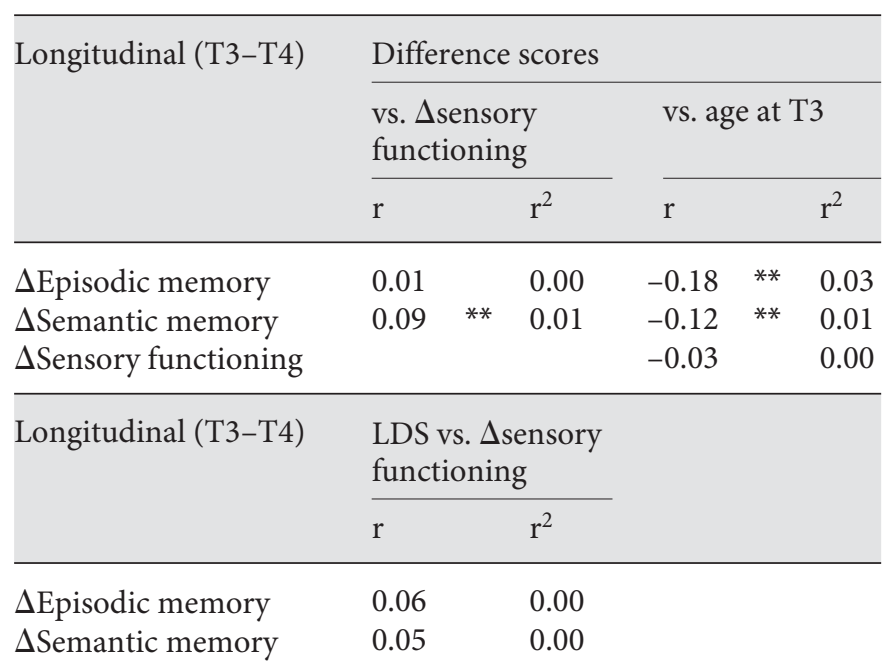

${ }^{* *} \mathrm{p}<0.01$ (two-tailed).

age. Thus, the NAC sample correlations (weighted averages) were approximately half of the correlations in the age-heterogeneous sample. The explained variances for the weighted average were only about $2 \%$, which means that as much as $98 \%$ of the variations in episodic and semantic memory scores remained to be explained by other factors than between-person variation in sensory functioning.

Since there is no variation in the age variable in the NAC design, shared age-related variance cannot be calculated between the cognitive and sensory variables. Instead, development in associations between cognitive and sensory ability across cohorts might reflect shared age-related variance [5]. An increase in the magnitude of correlations in older cohorts indicates shared age-related variance.

There was no steady increase in associations in older age cohorts over the examined age span. Rather, correlations varied across age cohorts, such that some cohorts showed strong and other weak associations. A fitted 
Table 5. Explained age-related variances in the age-heterogeneous sample

\begin{tabular}{lllll}
\hline Order of entered variables & $\begin{array}{l}\text { Cumu- } \\
\text { lative } \\
\mathrm{R}^{2}\end{array}$ & $\begin{array}{l}\text { Change } \\
\text { in } \\
\mathrm{R}^{2}\end{array}$ & $\begin{array}{l}\mathrm{F} \text { for } \\
\text { change }\end{array}$ & $\begin{array}{l}\text { Explained } \\
\text { age-related } \\
\text { variance }\end{array}$ \\
\hline $\begin{array}{l}\text { Cross-sectional (at T3) } \\
\text { Episodic memory }\end{array}$ & & & & \\
$\quad$ 1. Age & 0.312 & 0.312 & $478.86^{*}$ & \\
$\quad$ 1. Sensory functioning & 0.108 & 0.108 & $127.56^{*}$ & $29 \%$ \\
2. Age & 0.330 & 0.222 & $349.85^{*}$ & \\
$\begin{array}{l}\text { Semantic memory } \\
\text { 1. Age }\end{array}$ & 0.106 & 0.106 & $125.21^{*}$ & \\
$\quad$ 1. Sensory functioning & 0.069 & 0.069 & $78.29^{*}$ & $42 \%$ \\
$\quad$ 2. Age & 0.130 & 0.061 & $73.89^{*}$ & \\
\hline * p $<0.001$ (two-tailed). & & & & \\
\hline
\end{tabular}

trend line (of third order, $\mathrm{R}^{2}=0.47$ ) in figure 1 indicated that the correlations between episodic memory and sensory functioning across the examined age cohorts showed approximately a U-shaped curve. From a correlation of $\mathrm{r}=0.24$ at 45 years of age, the correlation trend curve decreases and does not increase again until older age cohorts (from approximately 70 years of age). The only age cohorts that demonstrated significant correlations in this study were $45,50,60,80$ and $85-90$ years of age. The correlations between semantic memory and the sensory variable showed almost the same pattern across age cohorts, with a peak at 80 years of age. The correlations between semantic memory and sensory functioning were significant in the two age cohorts that demonstrated the strongest significance for episodic memory, i.e. 45 and 80 years of age. Apart from the 80 years cohort, with an explained variation of $25-30 \%$, the other age cohorts showed very low levels of explained variation.

\section{Longitudinal Analyses}

Age-Heterogeneous Sample - Difference Scores

The correlations in the age-heterogeneous sample were much lower in the longitudinal (correlations between change scores across time) than in the cross-sectional study (correlations between absolute levels). Table 4 shows that the correlation between change in episodic memory ( $\Delta$ episodic memory) from $\mathrm{T} 3$ to $\mathrm{T} 4$ and sensory functioning ( $\Delta$ sensory functioning) during the same period was only $r=0.01$. The correlation between $\Delta$ semantic memory and $\Delta$ sensory functioning was high-
Table 6. Correlations and explained variance in the NAC samples (cross-sectional)

\begin{tabular}{|c|c|c|c|c|c|c|c|}
\hline \multirow[t]{2}{*}{ Age at T3 } & \multirow[t]{2}{*}{$\mathrm{n}$} & \multicolumn{3}{|c|}{$\begin{array}{l}\text { Episodic mem- } \\
\text { ory vs. sensory }\end{array}$} & \multicolumn{3}{|c|}{$\begin{array}{l}\text { Semantic mem- } \\
\text { ory vs. sensory }\end{array}$} \\
\hline & & $\mathrm{r}$ & & $r^{2}$ & $\mathrm{r}$ & & $\mathrm{r}^{2}$ \\
\hline 45 & 152 & 0.24 & $* *$ & 0.06 & 0.30 & $* *$ & 0.09 \\
\hline 50 & 154 & 0.17 & $*$ & 0.03 & 0.11 & & 0.01 \\
\hline 55 & 139 & -0.02 & & 0.00 & -0.07 & & 0.00 \\
\hline 60 & 160 & 0.20 & * & 0.04 & 0.17 & & 0.03 \\
\hline 65 & 136 & 0.04 & & 0.00 & -0.04 & & 0.00 \\
\hline 70 & 123 & 0.09 & & 0.01 & 0.17 & & 0.03 \\
\hline 75 & 91 & 0.04 & & 0.00 & 0.03 & & 0.00 \\
\hline 80 & 56 & 0.49 & $* * *$ & 0.25 & 0.52 & $* * *$ & 0.28 \\
\hline $85-90$ & 46 & 0.30 & $*$ & 0.09 & 0.19 & & 0.04 \\
\hline Weighted average & & 0.14 & & 0.02 & 0.13 & & 0.02 \\
\hline
\end{tabular}

er $(r=0.09)$, but still weak. This means that the variation in 5 -year change in sensory functioning explains very little of the variation in 5-year change in episodic and semantic memory $\left(r^{2}=0 \%\right.$ and $r^{2}=1 \%$, respectively). In table 4 , age at T3 is used as an age indicator, but note that this is not a change variable. All participants were followed during a 5-year follow-up period, which makes age change a constant, and this is also why no calculation of explained age-related variance was done for the longitudinal dataset.

Age-Heterogeneous Sample - LDS

Two bivariate LDS models were used, one for episodic memory and sensory functioning, and one for semantic memory and sensory functioning. As can be seen in table 4 , the correlations between LDS in the age-heterogeneous sample were approximately on the same low level for both $\Delta$ episodic memory $-\Delta$ sensory functioning, and for $\Delta$ semantic memory $-\Delta$ sensory functioning $(r=0.06$, $\mathrm{t}=0.60$, n.s., and $\mathrm{r}=0.05, \mathrm{t}=0.55$, n.s., respectively). These weak correlations and explained variances were much lower than in the cross-sectional study, and this result pattern is very similar to the one with difference scores. The model fit was good according to the probability that the root mean square error of approximation was below 0.05 , since the probability was $97 \%$ for the episodic-sensory model and $94 \%$ for the semantic-sensory model. 


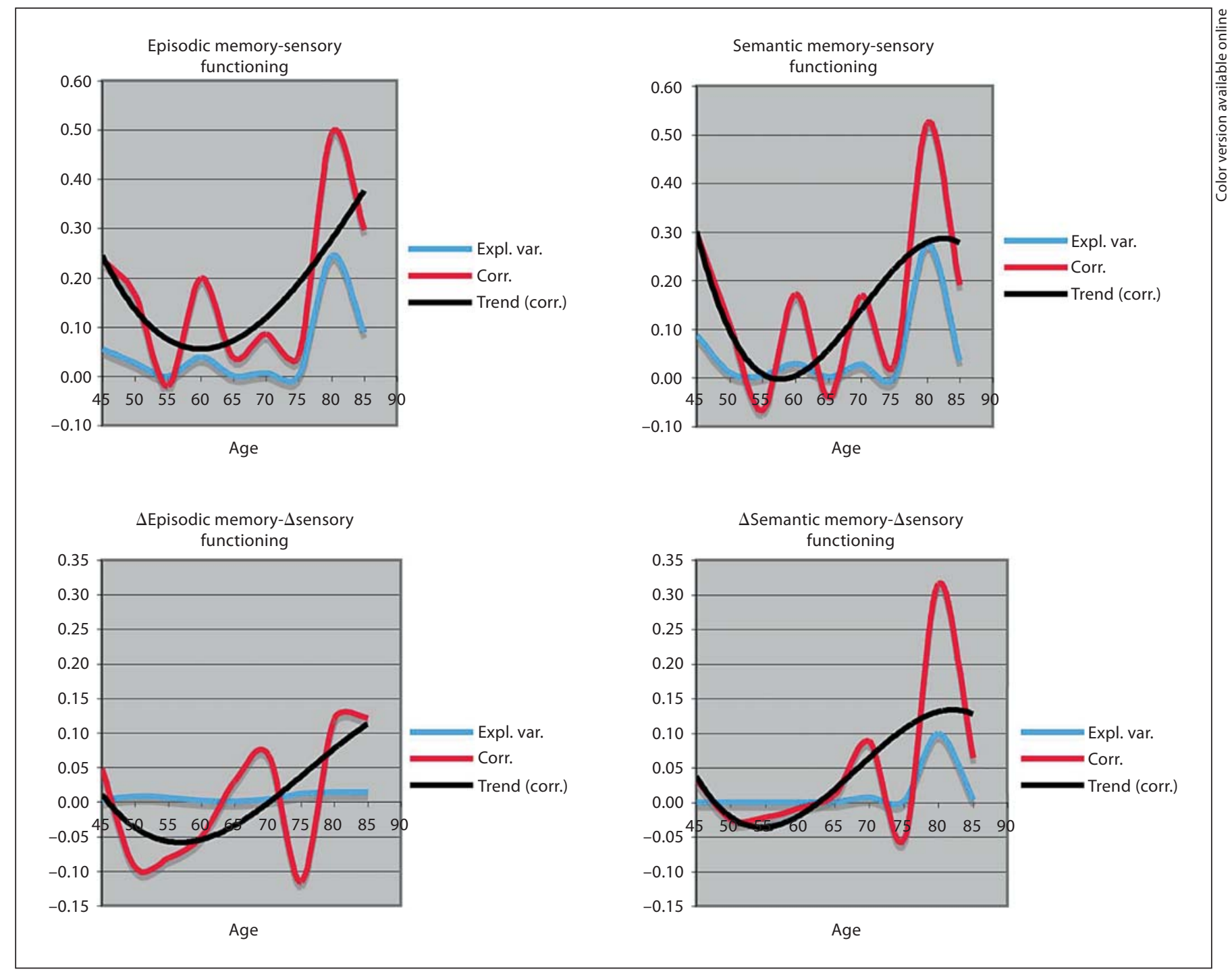

Fig. 1. Correlations and explained variances across age cohorts.

\section{NAC Samples - Difference Scores}

The correlations between $\Delta$ episodic memory and $\Delta$ sensory functioning fluctuated between $\mathrm{r}=-0.11$ and $r=0.12$, with a weighted average of $r=-0.01$ (see table $7)$. The amount of variance in $\Delta$ episodic memory accounted for by $\Delta$ sensory functioning varied between $0 \%$ and $1 \%$. According to these figures, the variation in 5 year change in episodic memory is not explained by the variation in 5-year change of sensory functioning. No steady increase in associations across cohorts was found. As for the cross-sectional NAC design, the correlation trend curve (of third order, $\mathrm{R}^{2}=0.42$ ) across age cohorts showed a U-shaped pattern (see fig. 1), with a peak for change between 80 and 85 years of age. Note that many of the age cohorts showed negative correlations between $\Delta$ episodic memory and $\Delta$ sensory functioning, which indicate that these two variables, for certain age groups, might go in opposite directions, i.e. $\Delta$ sensory functioning decreases when $\Delta$ episodic memory increases. Approximately the same tendency found for $\Delta$ episodic memory was also found for $\Delta$ semantic memory, although the peak between 80 and 85 was stronger. However, the only age cohort correlation that was significant in the present longitudinal NAC design, was in fact this peak (between 80 and 85 years of age). 
Table 7. Correlations and explained variance in the NAC samples (longitudinal)

\begin{tabular}{|c|c|c|c|c|c|c|}
\hline \multirow[t]{2}{*}{$\begin{array}{l}\text { Age } \\
\text { at T3 }\end{array}$} & \multirow[t]{2}{*}{$\begin{array}{l}\text { Age } \\
\text { at T4 }\end{array}$} & \multirow[t]{2}{*}{$\mathrm{n}$} & \multicolumn{2}{|c|}{$\begin{array}{l}\Delta \text { Episodic } \\
\text { memory vs. } \\
\Delta \text { sensory funct. }\end{array}$} & \multicolumn{2}{|c|}{$\begin{array}{l}\Delta \text { Semantic } \\
\text { memory vs. } \\
\Delta \text { sensory funct. }\end{array}$} \\
\hline & & & r & $\mathrm{r}^{2}$ & $\mathrm{r}$ & $r^{2}$ \\
\hline 45 & 50 & 152 & 0.05 & 0.00 & 0.03 & 0.00 \\
\hline 50 & 55 & 154 & -0.09 & 0.01 & -0.02 & 0.00 \\
\hline 55 & 60 & 139 & -0.08 & 0.01 & -0.02 & 0.00 \\
\hline 60 & 65 & 160 & -0.05 & 0.00 & -0.01 & 0.01 \\
\hline 65 & 70 & 136 & 0.03 & 0.00 & 0.01 & 0.00 \\
\hline 70 & 75 & 123 & 0.07 & 0.00 & 0.09 & 0.01 \\
\hline 75 & 80 & 91 & -0.11 & 0.01 & -0.05 & 0.00 \\
\hline 80 & 85 & 56 & 0.12 & 0.01 & $0.32^{*}$ & 0.10 \\
\hline 85-90 & $90-95$ & 46 & 0.12 & 0.01 & 0.06 & 0.00 \\
\hline \multicolumn{3}{|c|}{ Weighted average } & -0.01 & 0.00 & 0.02 & 0.00 \\
\hline
\end{tabular}

${ }^{*} \mathrm{p}<0.05$ (two-tailed).

\section{Discussion}

We examined the common cause account within a longitudinal NAC design. The common cause account is based on the assumption that sensory/sensorimotor functioning is mediating the association between chronological aging and cognition $[1,4]$. This mediating effect is interpreted in the common cause account as a third factor that affects both sensory/sensorimotor and cognitive functioning, and possibly the central nervous system [1]. Importantly, the common cause account was developed based on cross-sectional data, which are especially biased by cohort effects and effects related to mean trends $[5,7]$. However, these effects can be controlled for in NAC and in longitudinal studies. There are only a few NAC studies, but none have found convincing support for the common cause account $[5,12]$, and the results from longitudinal studies are complex and not consistent [12-15]. The present study is unique in the sense that it combines a sequential NAC design with a longitudinal design to validate the common cause account.

The shared variances between sensory and cognitive abilities in the age-heterogeneous sample (in the crosssectional design) were lower than what Baltes and Lindenberger $[1,4]$ found in their original studies. They found a shared variance of $11 \%$ between intelligence (assessed by 14 different cognitive tests) and sensory functioning for a young sample (25-69 years of age), and 31\% for an old sample (70-103 years of age). In the total age- heterogeneous sample (45-90 years of age) in the present study, the figures were, however, $11 \%$ for episodic and $7 \%$ for semantic memory versus sensory abilities. The correlations for episodic memory (a fluid ability) in the present study were approximately at the same level as for semantic memory (a crystallized ability). In the study by Baltes and Lindenberger [4], higher correlations were found only for perceptual speed (a fluid ability), otherwise Baltes and Lindenberger also found similarly sized correlations for both fluid and crystallized abilities.

The present study demonstrated much lower correlations and explained variances in the NAC sample analyses compared to the age-heterogeneous sample (mainly in the cross-sectional design). This is also in line with our first hypothesis and also in agreement with what other studies have found (e.g. [5]). However, the longitudinal design showed very low correlations and explained variances for both the age-heterogeneous (in both analyses with difference scores and with LDS) and age-homogeneous samples.

The cross-sectional data demonstrated much stronger sensory-memory associations than the longitudinal data. This was the case both for the age-heterogeneous (with difference scores and with LDS) and age-homogeneous samples, which was in accordance with the second hypothesis. Previous longitudinal research is not as consistent as cross-sectional, and gives only partial support for the common cause account [12-15].

According to the third hypothesis, the correlations were expected to be stronger in older cohorts than in younger cohorts. However, the tendency, over the examined age span for both episodic and semantic memory versus sensory functioning, was more of a U-shaped correlation curve with a rising trend only in the oldest age cohorts, which is mainly because of a peak at 80 years of age. This peak might be due to a simultaneous decrease in health status, but the focus of the present study was not to study health variables. It would, however, be of interest to examine this further in future studies. This trend was found both for the cross-sectional and longitudinal design (even if the explained variances were very low in the longitudinal design). Another possible explanation for the U-shaped trend curve might be selection bias (i.e. initial selection in the project and attrition in the oldest age cohorts), which is a confounder in the NAC design [8].

The findings of the present study support the notion that the common cause is mainly valid for old and very old people $[1,4]$, even if the explained variances in the present study were rather low. According to the present findings, it seems that the common cause account only 
explains variations in older age groups, but still provides a rather weak explanation. Christensen and Mackinnon [12] also suggested, based on a cross-sectional NAC design with three cohorts, the possibility that the common cause account is not valid for middle age or younger old age. In the present study, younger age cohorts demonstrated a relation in the opposite direction to what the common cause predicts. The findings also indicate that the development might proceed in steps and not continuously as the trend curve suggests. The division in the present study into separate age cohorts made it possible to see this development of the relationship between sensory and cognitive variables across age rather specified, which is not possible with broader age groups, as in the Baltes and Lindenberger study [4] of two age groups (2569 and $70-103$ years of age).

The findings of the present study are unlikely to be caused by smaller variations in the different age cohorts compared to the age-heterogeneous sample. A study of the variation showed that even in the age cohorts, the standard deviations were approximately at the same level as in the total sample, and the coefficient of variation (measured as standard deviation divided by the mean) was also similar for the age cohorts compared to the ageheterogeneous sample.

Although the present study has a strong design, there are some limiting aspects of the study that should be recognized. There were fewer participants in the oldest age cohorts, and the attrition effects are difficult to estimate. The practice effects in Betula have been estimated on a general level [26], but these estimations are difficult to adapt to a within-person analysis since these estimations are based on group levels instead of individual levels, and in this case with different variables and age groups. The choice of indicators included in the sensory composite might have importance for the outcome, and other sensory measures might have shown a slightly different picture. This type of longitudinal NAC study should therefore be replicated with other sensory/ sensorimotor measures, and the Betula project will provide more detailed sensory/sensorimotor measures in the future. The low explained variances obtained in the longitudinal design might be a result of the follow-up period (5 years) being too short to indicate enough change. It would be interesting to test the common cause account with a longer follow-up period, and Betula will have this possibility in the future. Since many of the correlations in the NAC design were rather low, probably more participants in some of the cohorts would have been needed to reach a level of significance. Now, only associations in some of the cohorts reached significance. However, even with more participants, it would have resulted in low correlation levels and low explained variances.

\section{Conclusions}

The correlations and explained variances were considerably lower in the age-homogeneous samples compared to the age-heterogeneous sample. It was also lower in the longitudinal compared to the cross-sectional design. When combining these, the associations were almost eliminated. This suggests the link between age-related sensory and cognitive change is very weak over the examined life span. Further, the associations seemed to change direction over the examined life span, although there was a tendency that the associations became stronger in older cohorts, which indicates age-related associations [5]. We found minor support for the common cause in the older cohorts, mainly in the cross-sectional NAC analyses. The correlations tended to become stronger in older samples, but only for certain cohorts (e.g. the 80years cohort) and not so continuously as a trend curve might show. However, the present study did not provide any support for the common cause as a major source of individual differences in sensory and memory functioning. Overall the explained variances were low, which leads to the conclusion that the common cause account has a low explanatory value. Since the link between sensory and cognitive functioning in the present study was weak, other mediating accounts (e.g. the sensory deprivation account) involving a relationship between sensory and cognitive functioning would also have a problem with low explanatory value. Better explanation models are needed, perhaps reviewing the mixture of candidates. There are other candidate mediators, e.g. dopamine system [27] and neural noise [28]. Instead of seeking a candidate to be the only explanation, it could be that several factors interact in a way that leads to the outcomes in aging. The study of higher-order interaction effects in a longitudinal setting would be an interesting way to go, and could include both genes and health variables [29]. Towards this end, Li and Lindenberger [13] and Lindenberger and Ghisletta [30] suggest this common cause might not be the only reason behind agechanges in sensory/sensorimotor and cognitive functions. Probably a more multidimensional model of explanation is needed. 


\section{Acknowledgements}

The Betula Longitudinal Project is supported by the Bank of Sweden Tercentenary Foundation (1988-0082:17), Swedish Council for Planning and Coordination of Research (D19880092, D1989-0115, D1990-0074, D1991-0258, D1992-0143, D1997-0756, D1997-1841, D1999-0739 and B1999-474), Swedish Council for Research in the Humanities and Social Sciences
(F377/1988-2000), the Swedish Council for Social Research (1988-1990:88-0082, and 311/1991-2000) and the Swedish Research Council (345-2003-3883, 315-2004-6977). Also, we acknowledge the contribution of the staff in the Betula project. A.W. was funded by a grant from Swedish Council for Research in the Humanities and Social Sciences (Dnr 421-2002-2575). O.S. was supported by a grant from the Royal Swedish Academy of Sciences.

\section{References}

1 Lindenberger U, Baltes PB: Sensory functioning and intelligence in old age: a strong connection. Psychol Aging 1994;9:339-355.

-2 Luszcz MA, Bryan J: Toward understanding age-related memory loss in late adulthood. Gerontology 1999;45:2-9.

-3 Lindenberger U, Baltes PB: Intellectual functioning in old and very old age: cross-sectional results from the Berlin Aging Study. Psychol Aging 1997;12:410-432.

4 Baltes PB, Lindenberger U: Emergence of a powerful connection between sensory and cognitive functions across the adult life span: a new window to the study of cognitive aging? Psychol Aging 1997;12:12-21.

5 Hofer SM, Berg S, Era P: Evaluating the interdependence of aging-related changes in visual and auditory acuity, balance, and cognitive functioning. Psychol Aging 2003; 18: 285-305.

-6 Anstey KJ, Smith GA: Interrelationships among biological markers of aging, health, activity, acculturation, and cognitive performance in late adulthood. Psychol Aging 1999;14:605-618.

7 Hofer SM, Flaherty BP, Hoffman L: Crosssectional analysis of time-dependent data: mean-induced association in age-heterogeneous samples and an alternative method based on sequential narrow age cohort samples. Multivariate Behav Res 2006;41:165187.

-8 Hofer SM, Sliwinski MJ: Understanding ageing. An evaluation of research designs for assessing the interdependence of ageing-related changes. Gerontology 2001;47:341-352.

-9 Sternäng O, Wahlin Å, Nilsson LG: Examination of the processing speed account in a population-based longitudinal study with narrow age cohort design. Scand J Psychol 2008;49:419-428.

10 Tay T, Wang JJ, Kifley A, Lindley R, Newall P, Mitchell P: Sensory and cognitive associations in older persons: Findings from an older Australian population. Gerontology 2005; 52:386-394.
11 Bäckman L, Small BJ, Wahlin Å, Larsson M: Cognitive functioning in very old age; in Craik FIM, Salthouse TA (eds): The Handbook of Aging and Cognition. Mahwah, Lawrence Erlbaum Associates, 2000, pp 499-558.

12 Christensen H, Mackinnon A: Exploring the relationships between sensory and cognitive functions across the adult life span; in Dixon RA, Bäckman L, Nilsson LG (eds): New Frontiers in Cognitive Aging. Oxford, Oxford University Press, 2004, pp 217-234.

13 Li KZ, Lindenberger U: Relations between aging sensory/sensorimotor and cognitive functions. Neurosci Biobehav Rev 2002;26: 777-783.

14 Lövdén $\mathrm{M}$, Ghisletta $\mathrm{P}$, Lindenberger U: Cognition in the Berlin Aging Study (BASE): the first 10 years. Aging Neuropsychol Cogn 2004;11:104-133.

15 Anstey KJ, Hofer SM, Luszcz MA: A latent growth curve analysis of late-life sensory and cognitive function over 8 years: evidence for specific and common factors underlying change. Psychol Aging 2003;18:714-726.

16 Nilsson LG, Bäckman L, Erngrund K, Nyberg L, Adolfsson R, Bucht G, Karlsson S, Widing M, Winblad B: The Betula prospective cohort study: memory, health, and aging. Aging Neuropsychol Cogn 1997;4:1-32.

-17 Nilsson LG, Adolfsson R, Bäckman L, de Frias CM, Molander B, Nyberg L: Betula: A prospective cohort study on memory, health, and aging. Aging Neuropsychol Cogn 2004; 11:134-148.

18 Runge PE: Eduard Jaeger's Test-Types (Schrift-Scalen) and the historical development of vision tests. Trans Am Ophthalmol Soc 2000;98:375-438.

19 Koike KJ, Hurst MK, Wetmore SJ: Correlation between the American Academy of Otolaryngology-Head and Neck Surgery fiveminute hearing test and standard audiologic data. Otolaryngol Head Neck Surg 1994;111: 625-632.

20 Tabachnik BG, Fidell LS: Using Multivariate Statistics,. ed 4. Needham Heights, Allyn \& Bacon, 2001.
21 Lindenberger U, Pötter U: The complex nature of unique and shared effects in hierarchical linear regression: implications for developmental psychology. Psychol Meth 1998; 3:218-230.

22 McArdle JJ: A latent difference score approach to longitudinal dynamic structural analysis; in Cudeck R, Du Toit S, Sörbom D (eds): Structural Equation Modeling: Present and Future. A Festschrift in Honor of Karl Jöreskog. Lincolnwood, Scientific Software Int., 2001, pp 341-380.

23 McArdle JJ: Latent variable modeling of differences and changes with longitudinal data. Annu Rev Psychol 2009;60:577-605.

24 McArdle JJ, Hamagami F: Latent difference score structural models for linear dynamic analyses with incomplete longitudinal data; in Collins LM, Sayer AG (eds): New Methods for the Analyses of Change. Washington, Am Psychol Assoc, 2001, pp 137-175.

25 Steiger JH: Structural model evaluation and modification: an interval estimation approach. Multivariate Behav Res 1990;25: 173-180.

26 Rönnlund M, Nyberg L, Bäckman L, Nilsson LG: Stability, growth, and decline in adult life span development of declarative memory: cross-sectional and longitudinal data from a population-based study. Psychol Aging 2005;20:3-18.

27 Bäckman L, Ginovart N, Dixon RA, Wahlin TB, Wahlin Å, Halldin C, Farde L: Age-related cognitive deficits mediated by changes in the striatal dopamine system. Am J Psychiatry 2000;157:635-637.

28 Welford AT: Signal, noise, performance, and age. Hum Factors 1981;23:97-109.

-29 Sternäng O, Wahlin $\AA$, Adolfsson R, Sleegers K, Van Broeckhoven C, Nilsson LG: APOE and lipid level synergy effects on declarative memory functioning in adulthood. Eur Psychol 2009;14:268-278.

30 Lindenberger U, Ghisletta P: Cognitive and sensory declines in old age: gauging the evidence for a common cause. Psychol Aging 2009;24:1-16. 